Article

\title{
A Closed-Form Technique for the Reliability and Risk Assessment of Wind Turbine Systems
}

\author{
Akwasi F. Mensah * and Leonardo Dueñas-Osorio \\ Department of Civil and Environmental Engineering, Rice University, Houston, TX 77251-1892, USA; \\ E-Mail: Leonardo.duenas-osorio@rice.edu \\ * Author to whom correspondence should be addressed; E-Mail: afm3@rice.edu; \\ Tel.: +1-713-348-5292; Fax: +1-713-348-5268.
}

Received: 5 April 2012; in revised form: 25 May 2012 / Accepted: 29 May 2012 / Published: 4 June 2012

\begin{abstract}
This paper proposes a closed-form method to evaluate wind turbine system reliability and associated failure consequences. Monte Carlo simulation, a widely used approach for system reliability assessment, usually requires large numbers of computational experiments, while existing analytical methods are limited to simple system event configurations with a focus on average values of reliability metrics. By analyzing a wind turbine system and its components in a combinatorial yet computationally efficient form, the proposed approach provides an entire probability distribution of system failure that contains all possible configurations of component failure and survival events. The approach is also capable of handling unique component attributes such as downtime and repair cost needed for risk estimations, and enables sensitivity analysis for quantifying the criticality of individual components to wind turbine system reliability. Applications of the technique are illustrated by assessing the reliability of a 12-subassembly turbine system. In addition, component downtimes and repair costs of components are embedded in the formulation to compute expected annual wind turbine unavailability and repair cost probabilities, and component importance metrics useful for maintenance planning and research prioritization. Furthermore, this paper introduces a recursive solution to closed-form method and applies this to a 45-component turbine system. The proposed approach proves to be computationally efficient and yields vital reliability information that could be readily used by wind farm stakeholders for decision making and risk management.
\end{abstract}

Keywords: wind turbine; system reliability; closed-form solutions; consequence analysis; risk 


\section{Introduction}

This study introduces a closed-form method for analyzing the system-level reliability of wind turbines for any desired system failure event definition. Combinatorial and recursive approaches of the method are proposed to efficiently and tractably tackle system reliability assessments of wind turbines of different system size. The study also improves the method to assess system-level risk and to explicitly compute component importance measures to identify critical components leading to system-level failures.

Wind energy is the fastest growing renewable energy, with its total energy share increasing at a rapid average annual rate of $32 \%$ in the past decade $[1,2]$. To sustain its growth and viability in the highly competitive power industry, the industry must not only afford the most productive and economic configurations of wind turbines, but also highly reliable power generation. Reliability of a wind turbine refers to the ability of the system to perform its intended function under prescribed conditions for a specific period of time. Reliability has a significant impact on the costs and benefits of wind turbine projects [3]. It is an important metric to judge the long-term performance and operational cost of evolving wind turbine products. Governments, policy makers and financial institutions, among other things, rely on reliability information to evaluate the viability of wind farm projects. Reliability analyses that provide failure probability estimates of a system are important element of the process for making decisions on system design, retrofit, maintenance, operation and repair.

The reliability of a wind turbine as in the case of typical engineered complex systems is dependent on the reliability of its constituent subsystems or components. Therefore, the state of the system at a given time can be described by a Boolean or logical function of failure or survival of its components. Analyzing the reliability of complex systems is generally not an easy task and occasionally infeasible due to the large numbers of components involved or incompleteness of information available [4]. Monte Carlo simulation (MCS) is a widely employed method in many infrastructure reliability studies [5-7]. MCS provides good approximations and is capable of handling multiple system-specific modeling conditions, correlations among events and different system failure event definitions. However, the simulation approach has high computational complexity which increases as the number of components grows [8]. Existing system reliability methods such as first order reliability method have minimal flexibility to accommodate various types and amounts of information, and therefore their applications are limited to providing probability bounds for series and parallel systems [4]. Analytical or closed-form techniques are emerging as alternatives to numerical simulations because of their flexibility, insight and potential efficiency in evaluating system reliability. These approaches are tractable with explicit input-output relationships between component and system failures, thus allowing for sensitivity analyses and easy determination of component importance measures. One such example is the combinatorial non-recursive approach used for bridge systems by Dueñas-Osorio and Padgett [9]. Another such example is the matrix-based system reliability (MSR) method [10].

In the wind industry, a number of published works focus on reliability models of components of the wind turbine with fewer studies assessing the reliability of an entire wind turbine system. Earlier studies (e.g., [11]) model wind as a stochastic process and combine it with the power-speed curve to determine the system-level reliability of wind turbines [12]. Other studies have modeled wind turbines as part of a large power system instead of isolated systems. These studies directly focus on 
system-level reliability, while ignoring the effect of assembly or component level reliability. To incorporate component reliabilities, Markov modeling [12], failure modes and effects analysis (FMEA) [13], pre-posterior Bayesian decision theory [14] and fault-tree analysis [15] are techniques which have been adopted in recent times to assess wind turbine reliability. A major drawback to the implementation of these methods is that they are computationally inefficient or infeasible at times for systems with large sizes (number of components). Additionally, most of these methods become more complex when attempting to account for correlation among components and trying to handle varying consequence units of component failures. Hence, developments in lifeline system reliability that are flexible and computationally efficient can be adapted to wind turbines and overcome some of the noted difficulties. Recently, Dueñas-Osorio and Rojo [8] proposed an analytical approach in a combinatorial form for obtaining the probability distribution of a customer service availability metric for generic radial networks. They also present a recursive solution to this system reliability technique that considers all possible $2^{N}$ system failure events in efficient computational time $O\left(N^{2}\right)$ for a system with $N$ components. They further allow the possibility to account for correlation, although through reliability bounds.

The current study explores the use of the combinatorial system reliability method to compute wind turbine failure probabilities for different failure event definitions. The paper also improves the method to quantitatively incorporate consequences of component failures for system-level risk assessment. Furthermore, this study exploits the flexibility and traceability of the combinatorial technique to explicitly quantify importance measures that indicate the criticality of individual components to the reliability of a wind turbine. To illustrate the application of the combinatorial non-recursive method, this research uses a 12-subassembly wind turbine system and evaluates the probability mass function of the system failure considering all possible configurations of subassembly failure and survival events. Crude MCS is also conducted and the results are compared to the closed-form probability distribution of system. Additionally, consequence analysis is performed to determine annual probabilities of system unavailability by integrating the unique subassembly downtimes in the reliability formulation. Cost-based reliability analysis based on costs of repairing, replacing or rebuilding subassemblies is also included in this paper to further illustrate the flexibility and scope of the method. Finally, the study uses the recursive version of the combinatorial closed-form method to evaluate failure probabilities needed to efficiently compute system reliability of a wind turbine system comprising of 45 components. This would not be possible with other analytical methods due to system size.

The paper is divided into five sections. The next section discusses the combinatorial and recursive forms of the closed-form system reliability solution. The third section contains an extension to the combinatorial solution for consequence or risk analysis. Component importance metrics are also introduced in this section and their computation framework. Applications of the two solutions to wind turbine systems are illustrated in the fourth section. Finally, the paper closes with conclusions, applications and highlights for future research. 


\section{Closed-Form Technique for Wind Turbine System Reliability Assessment}

Computing the system failure probability of a wind turbine is usually not an easy task since there is no clear definition on what constitutes system-level failure. There exists an entire set of system event possibilities such as failure of any key component, any two or any desired subset of wind turbine components resulting in system-level failure. The closed-form technique [8] is employed in this study to construct an entire probability mass function of the number of component failures from which system failure probabilities can be evaluated for any preferred system failure event definitions. The subsequent sections describe the combinatorial formulation and introduce the recursive algorithm to the closed-form solution.

\subsection{Combinatorial System Reliability}

Consider a wind turbine system made up of $N$ components each having two distinct states, failure and survival, and where the components are indexed by $I$, with $i=1,2, \ldots, N$. Then, any system event can be represented by an event vector $\boldsymbol{k}^{*}$ in the $\mathbb{R}^{N}$ space, with its entries defined as:

$$
k_{i}^{*}=\left\{\begin{array}{lc}
1 & \text { if } i^{\text {th }} \text { component fails } \\
0 & \text { otherwise }
\end{array}\right.
$$

Let the $2^{N}$ possible $\boldsymbol{k}^{*}$ vectors represent all possible combinations of component events leading to system events, and be assembled in a matrix $\boldsymbol{K}=\left\{k^{*} \in \mathbb{R}^{N}: k_{i}=1\right.$ or 0$\}$. Also, let $\boldsymbol{S}$ be a vector that contains all unique possible $s$ numbers of failed components. As an illustration, in a wind turbine system with twelve components, $\boldsymbol{S}=\{1,2, \ldots, 12\}$, the first and the last $\boldsymbol{k}^{*}$ vectors of the $\boldsymbol{K}_{12 \times 4096}$ matrix are [000000000000] and [111111111111], respectively, with the remaining 4094 vectors filled with combinations of zeros and ones. This arrangement characterizes a system with undefined system events and the failure probability is estimated either with definitions from the wind turbine industry or within bounds representing a series to parallel system definition. If the failure probability of the $i^{\text {th }}$ component is denoted as $P_{i}$, then, the probability of system failure as a result of exactly $s \in \boldsymbol{S}$ number of components failing is given by:

$$
P_{s y s}(s)=\sum_{\boldsymbol{k}^{*} \in \boldsymbol{K}_{\boldsymbol{s}}} \prod_{i=1}^{N} P_{i}^{k_{i}}\left(1-P_{i}\right)^{1-k_{i}}
$$

where $\left(1-P_{i}\right)$ is the reliability of the $i^{\text {th }}$ component and $\boldsymbol{K}_{\boldsymbol{s}}$ is a subset of $\boldsymbol{K}$ containing all $\boldsymbol{k}^{*}$ vectors whose sum equals $S$. A probability mass function of the system failure can be obtained by evaluating Equation (1) for all $\boldsymbol{k}^{*} \in \boldsymbol{K}_{\boldsymbol{s}}$ and then for all integers $s \in \boldsymbol{S}$.

This non-recursive solution offers important information about the probability of system failure and its relationship with component failures and can be used to obtain bounds when failure correlations are unknown. However, the non-recursive solution can be enhanced to account for correlation among component events in a similar fashion as shown for bridge systems [9], even though to the authors' knowledge, there is presently no correlation information publicly available for wind turbine systems and their components. Ongoing efforts to collect data by the Sandia National Laboratory [16] and Rice University [17], however, may yield valuable correlation structures in the future which would improve the accuracy of predictions by the closed-form method. The solution also has features which allow for 
further exploration and analysis of consequences beyond computing failure probabilities. However, the combinatorial routine involved in using the approach requires an exponentially increasing number of operations with $N$ as $O\left(2^{N}\right)$. This makes the application of the non-recursive formulation to wind turbines with a large number of documented components infeasible. The next section presents a recursive version of the closed-form method which is applicable to a wind turbine system with any number of components $N$.

\subsection{Recursive Solution to Combinatorial System Reliability}

Recursive methods allow complex formulae, such as Equation (2), to be solved efficiently by decomposing the expressions into smaller base forms. Techniques used for performing efficient evaluations of Possion-Binomial distributions in other fields such as survey sampling and survival analysis are employed to transform Equation (2) into a form which can be resolved in a recursive fashion. The system failure probability can be rewritten as:

$$
P_{\text {sys }}(s)=\sum_{\boldsymbol{k}^{*} \in \boldsymbol{K}_{\boldsymbol{s}}}\left(\prod_{i=1}^{N} \alpha_{i}^{k_{i}}\right) \prod_{i=1}^{N}\left(1+\alpha_{i}\right)^{-1}
$$

where $\alpha_{i}=\frac{P_{i}}{1-P_{i}}$. Since $\prod_{i=1}^{N}\left(1+\alpha_{i}\right)^{-1}$ is independent of the failure or survival of the $i^{\text {th }}$ wind turbine component in $\boldsymbol{k}^{*}$ and only dependent on the component failure probabilities $P_{i}$, it can be calculated beforehand. Hence, the computation load is only on the term $\sum_{\boldsymbol{k}^{*} \in \boldsymbol{K}_{\boldsymbol{s}}}\left(\prod_{i=1}^{N} \alpha_{i}^{k_{i}}\right)$ denoted by $Q\left(s, K_{s}\right)$. This $Q\left(s, K_{s}\right)$ term can be evaluated for each $s$ in a recursive manner. By defining $\boldsymbol{K}_{\boldsymbol{s}}(r)=\left\{\boldsymbol{k}^{*} \in \boldsymbol{K}_{\boldsymbol{s}}: k_{r}^{*}=0\right\}$ with $r=1$, although it can take any value from $\{1,2, \ldots N\}$ but the authors recommend the first term for tractability, this function can be expanded as:

$$
Q\left(s, K_{s}\right)=\sum_{\boldsymbol{k}^{*} \in \boldsymbol{K}_{\boldsymbol{s}}(r)}\left(\prod_{i=1}^{N} \alpha_{i}^{k_{i}}\right)+\alpha_{r} \sum_{\boldsymbol{k}^{*} \in \boldsymbol{K}_{\boldsymbol{s}-\mathbf{1}}(r)}\left(\prod_{i=1}^{N} \alpha_{i}^{k_{i}}\right)
$$

The recursive version of the closed-form solution is obtained by substituting Equation (4) into (3).

This formulation significantly reduces the time required to compute $P_{s y s}$ from an exponentially increasing $O\left(2^{N}\right)$ using the combinatorial non-recursive approach to a polynomial increasing time $O\left(N^{2}\right)$ as recently shown [18]. The recursive solution is an efficient technique to generate an entire probability mass density function for wind turbine systems with a large number of components. This is a prospective technique to handle risk or consequence analyses. Though such capability is already possible in the non-recursive approach, it is yet to be developed in the context of the recursive method.

\section{Closed-Form Technique Application to Wind Turbine Risk and Sensitivity Analyses}

Knowledge of the overall wind turbine failure probability, as typically reported in the wind industry, may not be readily useful for making financial, operational and maintenance decision especially when wind turbine components trigger varying consequences to the availability or performance of the wind turbines. An improvement to failure probability assessment is to compute the risk of system failure which incorporates unique attributes such as downtimes and repair costs of components to the failure probability. The combinatorial formulation for system approach presented in 
the previous section is improved to handle what is termed in this study as consequence-based reliability analysis. Additionally, metrics that provide a sound basis to judge the relative criticality of components are introduced as key by-products of the closed-form technique.

\subsection{Consequence-Based Reliability Analysis}

Let $q_{i}$ denote the number of hours lost (downtime) or repair cost as a consequence of the $i^{\text {th }}$ component failure and $\boldsymbol{q}^{*}$ is a vector in the $\mathbb{R}^{N}$ space containing $q_{i}$ elements. Let $\boldsymbol{C}$ be a vector containing all possible unique quantities of failure consequences $c$ such as component downtimes in $\mathrm{h}$ or repair costs in dollars. Each vector in $\boldsymbol{K}$ is multiplied by $\boldsymbol{q}^{*}$ to form $K_{C}=\left\{\boldsymbol{k}^{*} \in \boldsymbol{K}: \boldsymbol{k}^{*} \cdot \boldsymbol{q}^{*}=c\right\}$ representing all possible $k^{*}$ configurations that yield $c \in \boldsymbol{C}$ system failure consequence. Equation (2) is modified slightly to evaluate the wind turbine system risk, $P_{r s y s}$ for given $c$ units as shown below:

$$
P_{\text {rsys }}(c)=\sum_{\boldsymbol{k}^{*} \in \boldsymbol{K}_{\boldsymbol{C}}} \prod_{i=1}^{N} P_{i}^{k_{i}}\left(1-P_{i}\right)^{1-k_{i}}
$$

Like Equation (2), Equation (5) is evaluated for each $\boldsymbol{k}^{*} \in \boldsymbol{K}_{\boldsymbol{c}}$ and then for all integers $c \in \boldsymbol{C}$ to obtain a probability distribution of system risk or consequences.

\subsection{Component Criticality Analysis}

Typical fault tree analyses include importance measures which identify components that need to be maintained well so that the reliability of the system is not significantly reduced [19]. The closed-form technique can also provide the necessary information for computing importance measures. Two measures coined in this study as "component importance metric" and "component risk metric", are introduced in this study. The component importance metric (CIM) identifies critical components on the basis of their contribution to wind turbine reliability. This metric relies solely on the component failure information. CIM is useful for cases where information on consequence of component failure is unavailable or for systems with a large number of components which necessitate the recursive system reliability solution for computational feasibility. $C I M_{i}$ for component $i$ is the ratio of the system failure probability (i.e., failure of at least one component) to the joint probability of system failure and the survival of the $i^{\text {th }}$ component. It is expressed as:

$$
C I M_{i}=\frac{P_{\text {sys }}(s \geq 1)}{P_{\text {sys }}\left(s \geq 1, P_{i}=0\right)}
$$

The component risk metric (CRM) identifies components that are critical to the availability or repair cost of the wind turbine. This metric takes into account not only of the component failure probability, but also the resulting system risk. Consequently, $C R M$ provides an informative basis to rank components for design, monitoring and maintenance in order to maintain or improve the performance of the wind system as well as to estimate repair costs or downtimes. The importance metric for the $i^{\text {th }}$ component which relies on the system risk in Equation (5) is expressed as:

$$
C R M_{i}=\frac{P_{r s y s}(c \geq \tau)}{P_{r s y s}\left(c \geq \tau, P_{i}=0\right)}
$$


where $\tau$ is any length of system failure effects e.g., downtime, repair cost, etc., equal to or beyond a level for which the system risk is considered undesirable.

In both Equations (6) and (7), the system failure probability or risk in the denominator is evaluated by identifying the joint events in which $s$ or $c$ are exceeding and where the $i^{\text {th }}$ component does not fail. In this way, researchers, designers and engineers are able to identify the components whose reliabilities must be improved to increase the system reliability or reduce risks.

\section{Illustrative Examples}

This study utilizes two different datasets of available wind turbine failure information to demonstrate the application of the closed-form reliability technique to a simplified system comprising of 12 critical subassemblies, and a highly structured layout of a wind turbine system containing 45 components. The two datasets cover wind turbines of different population size, turbine size, farm sites and technologies. The source and relevant characteristics of the datasets are provided in the successive subsections. In both instances, a homogeneous Poisson process model, which assumes that the times between component failures are identical independent exponential random variables [20], is used to evaluate the failure probabilities of the components based on empirical failure rates. The model describing the probability $P_{i}(t)$ of having $n_{i}$ failures in time $t$ for component $i$ is given as:

$$
P_{i}\left(N(t)=n_{i}\right)=\frac{1}{n_{i} !}\left(\lambda_{i} t\right)^{n_{i}} e^{-\lambda_{i} t}
$$

where the failure rate $\lambda_{\mathrm{i}}$ of the $i^{\text {th }}$ component is the intensity function of the Poisson process. Failure rates are often determined using historical data and operational data recorded on wind farms or experimental testing of turbine components. In the absence of empirical data, expert opinions are sampled through surveys to estimate failure rates. The annual probability of at least one occurrence of the $i^{\text {th }}$ component failure is given by $P_{i}=1-e^{-\lambda}$.

The subsequent sections present the component failure data, analyses, results and discussions covering the two illustrative examples considered in this study. Probability distributions of system-level failure, annual wind turbine unavailability and expected annual cost of repair owing to system failure are constructed for the 12-subassembly wind turbine system. In addition, component criticality analyses (using Equations 6 and 7) are done to identify the importance of components with regards to the overall system annual failure, unavailability and recovery cost of the wind turbine.

\subsection{Example 1: 12-Subassembly Wind Turbine System}

This illustrative example utilizes failure information of 12 subassemblies of a wind turbine system. The subassemblies are the electrical subsystem, the rotor blades, the electrical controls, the yaw system, the generator, the hydraulic subsystem and the gear box. The rest are pitch control, air brakes, mechanical brake and main shaft. All other subassemblies are considered as a single unit in this taxonomy. The original failure data is collected from 158 to 653 onshore wind turbines of different models, ages, technologies, and manufactures in the region of Schleswig-Holstein in Osterrnfeld (Germany) by Landwitschafts-kammer (LWK) [21]. The data was collected for about 10 years, between 1993 and 2004, and approximately represents 5800 turbine years. This study uses summaries 
of the original data, as provided in Table 1, captured in terms of component failure rates and downtimes [22].

Table 1. Failure data for 12-subassembly wind turbine system [23].

\begin{tabular}{cccccc}
\hline Subassembly & $\boldsymbol{I}$ & $\begin{array}{c}\text { Failure Rate } \\
\text { (failures/yr/turbine) }\end{array}$ & $\begin{array}{c}\text { Downtime } \\
\text { (hours/failure) }\end{array}$ & \multicolumn{2}{c}{ Average cost (US\$/failure) } \\
Lower limit & Upper limit \\
\hline Electrical subsystem & 1 & 0.320 & 251 & 5,520 & 87,056 \\
Rotor or blades & 2 & 0.190 & 120 & 6,581 & 52,956 \\
Electrical controls & 3 & 0.239 & 60 & 440 & 6,000 \\
Yaw system & 4 & 0.116 & 58 & 401 & 9,121 \\
Generator & 5 & 0.139 & 161 & 332 & 53,228 \\
Hydraulic subsystem & 6 & 0.131 & 70 & 158 & 1,276 \\
Gear box & 7 & 0.134 & 345 & 1,476 & 153,601 \\
Pitch control & 8 & 0.083 & 65 & 2,087 & 17,832 \\
Air brakes & 9 & 0.040 & 105 & 3,076 & 3,076 \\
Mechanical brake & 10 & 0.055 & 48 & 200 & 1,483 \\
Main shaft & 11 & 0.031 & 135 & 4,318 & 15,668 \\
All others & 12 & 0.367 & 60 & 94,801 & 94,801 \\
\hline Total & & 1.846 & 1478 & 119,390 & 496,098 \\
Average & & 0.154 & 123 & 9,949 & 41,342 \\
COV * & 0.698 & 0.741 & 2.695 & 1.176 \\
\hline
\end{tabular}

* Coefficient of variation.

Also, Table 1 presents cost ranges for repairing, rebuilding or replacing subassemblies per failure. The costs are obtained from component repair costs provided in an operation and maintenance cost model developed for onshore wind turbines with capacity ranging from $750 \mathrm{~kW}$ to $2.4 \mathrm{MW}$ [23]. Prices in the model envelope turbine operations from 2004 to 2008, and do not consider catastrophic events, shipping and warehousing costs, repowering or retrofit works. The cost range covers the lowest to highest values of all components grouped under a subassembly. Furthermore, repair costs for the 1.5 MW rated turbine are used because this turbine is the largest size represented in the LWK data.

\subsubsection{System Failure Distribution}

In the non-recursive solution framework for this system (Equation 2), there is a total of $M=2^{12}=4096$ possible system state vectors $\boldsymbol{k}^{*}$ with each of the entries in $\left\{\begin{array}{ll}0 & 1\end{array}\right\}$ from which unique combinations of numbers of failed subassemblies are realized, i.e., $S=\{0,1, \ldots, 12\}$. As an illustration of the vectors that contribute to system failure, defined by at least one, two or three subassembly failures, the specific vector event when yaw system, the generator and the main shaft jointly fail is $\boldsymbol{k}^{*}=$ [000110000010]. The distribution of the failure probability as a function of the number of failed subassemblies is shown in Figure 1. It is observed that the annual reliability of the system $P_{\text {sys }}=P_{\text {sys }}(s=0)$ is 0.158 , if the wind turbine is considered to fail when at least a single subassembly fails. The wind turbine has an $84 \%$ chance of failure in any given year according to this definition. The annual likelihood of the system failing owning to at least two subassembly failures is $51 \%$. Interestingly, there is a negligible chance of wind turbine failure if at least six subassemblies have to fail before the system is considered as failed. Figure 2 compares the cumulative distribution functions 
(CDFs) of the system in terms of number of component failures evaluated using the closed-form technique and a Monte Carlo simulation routine consisting of 10,000 samples. The excellent agreement between the two CDFs confirms the adequate implementation of the exact closed-form combinatorial formulation.

Figure 1. Annual system failure probability mass function in terms of the number of subassembly failures.

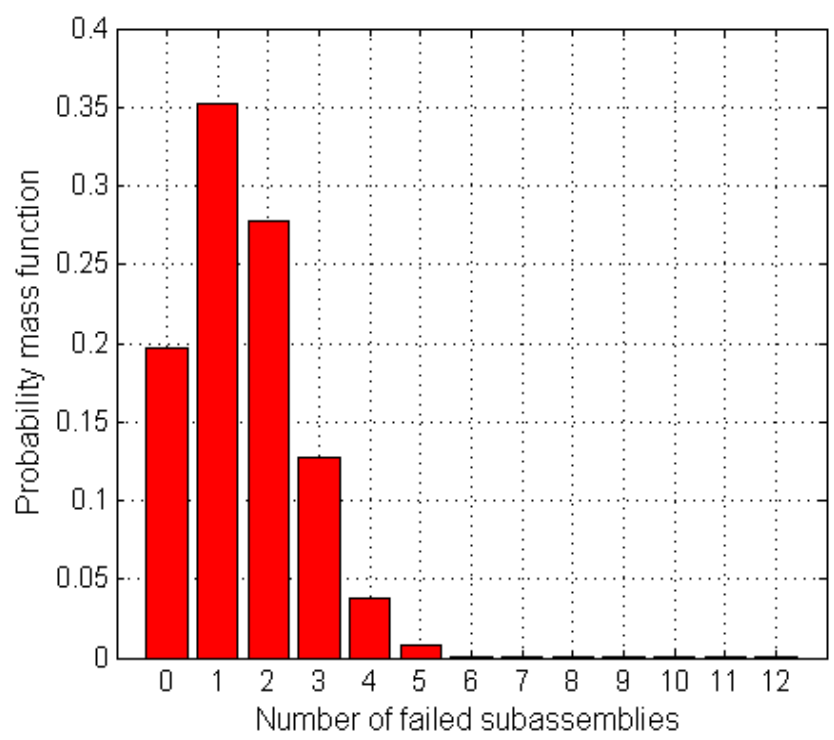

Figure 2. Comparison of annual system failure CDFs obtained by the closed-form technique and a naïve Monte Carlo simulation (MCS) approach.

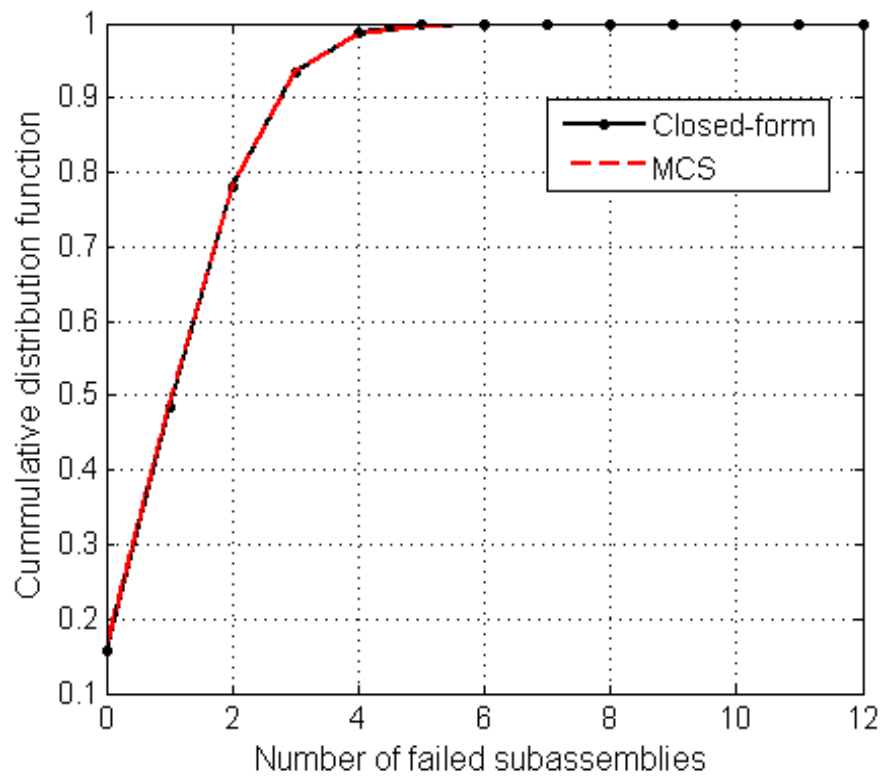

\subsubsection{Probability of System Unavailability}

The availability of wind turbines is directly related to the revenue generated from a wind farm. Understanding the expected annual availability or unavailability of a turbine is therefore essential for complete system performance assessment and annual revenue projections. The closed-form solution 
for consequence analysis is employed here to compute unavailability probabilities of the system by weighting failure probabilities of the subassemblies by their respectively induced average downtimes in hours per failure, as provided in Table 1. The downtime indicates the time needed to identify, access the plant, diagnose fault, mobilize labor and parts, replace or repair the component, and restore the wind turbine to full operation. The set of possibilities of wind turbine unavailability is given by $\boldsymbol{C}=\{0,48,58, \ldots, 1478\}$, in which 1478 is the total number of hours to be lost if all the 12 subassemblies fail and the numbers in between are realizable total numbers of hours given joint failures of different component subsets. Figure 3 shows the probability mass function of the wind turbine unavailability owing to component failures. The probability of the wind turbine being $100 \%$ available throughout an entire year is 0.158 , the same as the system reliability. However, there is a significant probability of $67 \%$ that a wind turbine is unavailable for at least 3 days $(72 \mathrm{~h})$. The likelihood of an unavailable wind turbine per failure for more than a week is $52 \%$. Furthermore, there is a $25 \%$ chance of losing more than 2 weeks of power generation owing to system failure. The probability of a wind turbine staying down for more than a month should failure occur is $2 \%$. The computed unavailability risks provide a framework for predicting expected annual number of stoppages, loss time, power generation and revenue loss per wind turbine, with little or no additional information required for such analysis.

Figure 3. Probability distribution of the 12-subassembly wind turbine unavailiability.

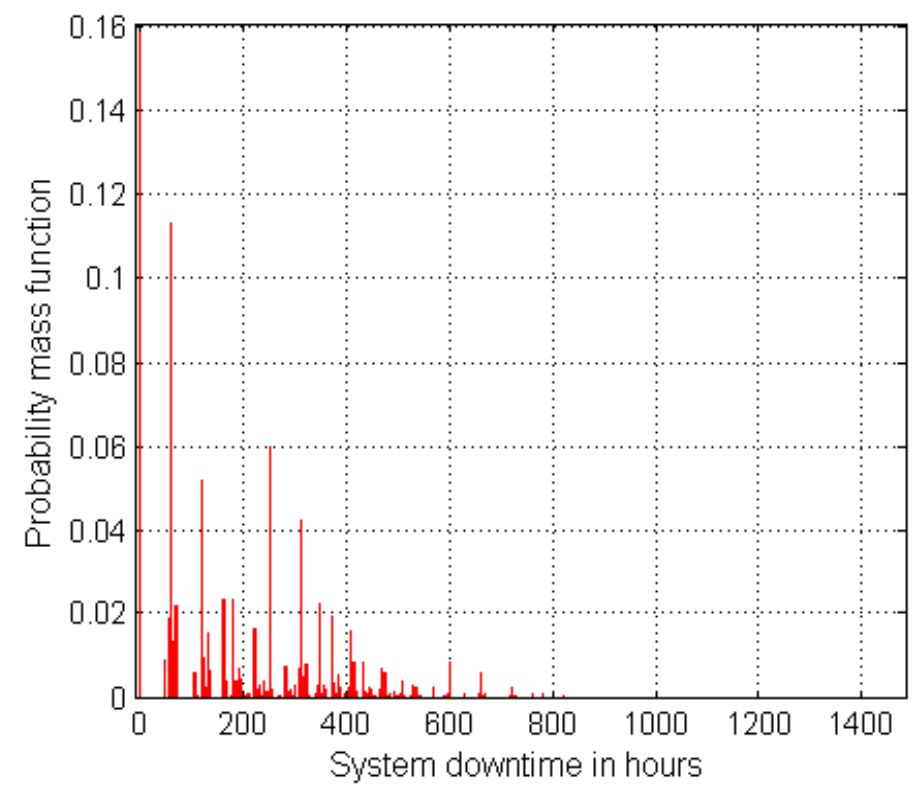

\subsubsection{System Repair Cost Probability}

Operation and maintenance (O\&M) contribute substantially to the production cost of wind turbines - up to $30 \%$ of energy cost [24]. Consequently, risk analysis in terms of repair cost is carried out to obtain information that is useful for O\&M decisions. The capacity of the closed-form technique to project repair cost for wind turbine is demonstrated in this section. The costs of repairing, replacing or rebuilding individual subassemblies are incorporated in Equation (5). Therefore, the system repair cost set of possibilities covers from US\$0 to US\$119,390 at the lower bound and US\$0 to almost US $\$ 500,000$ as the upper limit. Figure 4 shows the distributions of repair cost risk for the 
12-subassembly wind turbine system with the repair cost for the lower and upper limits in the primary and secondary X-axis, respectively. As expected, there is a $15.8 \%$ chance that no cost is incurred on repairing the turbine system in any year. In both probability mass functions for the low and high cost estimates, the probabilities are concentrated at lower repair cost values. For instance on the lower cost bound, the likelihood of exceeding $\$ 1,000$ in annual repair expenditure for a single turbine is $69 \%$ while the annual risk of at least $\$ 10,000$ in system repair cost is $35 \%$. There are relatively insignificant probabilities of realizing repair costs between $\$ 12,000$ and $\$ 94,000$. The spikes observed around $\$ 100,000$ are a result of repair cost contribution from the 'all others' subassembly which also has the highest failure rate. The system has a $12 \%$ probability of needing repairs costing more than $\$ 100,000$ on the lower limit. In the case of the upper component cost limits, the risk of exceeding system repair cost of $\$ 10,000$ is $73 \%$. There is $38 \%$ chance of spending beyond $\$ 100,000$ to repair a wind turbine. There is a negligible possibility of attaining the maximum repair costs in both cases.

Figure 4. Probability distribution of repair cost for 12-subassembly wind turbine.

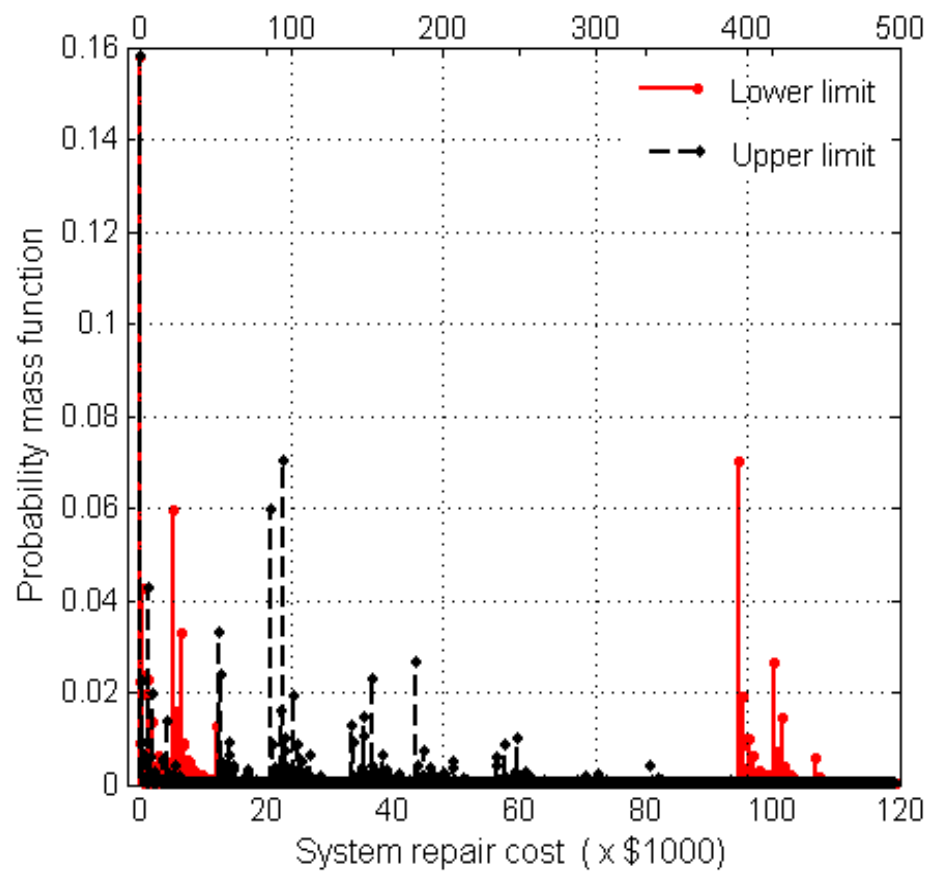

\subsubsection{Component Importance Metrics}

To further illustrate the tractability of the closed-form technique on the 12-subassembly wind turbine, Table 2 provides the set of component importance metrics (CIMs) and component risk metrics (CRMs) of the subassemblies. Two sets of CRMs are determined in this study: the first based on the annual turbine unavailability probabilities and the second based on annual turbine repair costs. For the purposes of differentiating between the two, these metrics are referred to as component availability metric (CAM) and component cost metric (CCM), respectively. The subassemblies are ranked from the most critical subassembly (Rank \#1) to the least critical subassembly according to these metrics. 
Table 2. Subassembly importance measures and ranks.

\begin{tabular}{lcccccc}
\hline Subassembly & CIM & Rank & CAM & Rank & CCM & Rank \\
\hline Electrical system & 1.076 & 2 & 1.239 & 1 & 1.259 & 2 \\
Rotor or blades & 1.041 & 4 & 1.122 & 2 & 1.183 & 3 \\
Electrical controls & 1.053 & 3 & 1.061 & 6 & 1.087 & 9 \\
Yaw system & 1.024 & 8 & 1.034 & 8 & 1.097 & 7 \\
Generator & 1.029 & 5 & 1.085 & 3 & 1.153 & 4 \\
Hydraulic system & 1.027 & 7 & 1.037 & 7 & 1.083 & 10 \\
Gear box & 1.028 & 6 & 1.082 & 4 & 1.150 & 5 \\
Pitch control & 1.016 & 9 & 1.025 & 9 & 1.122 & 6 \\
Air brakes & 1.008 & 11 & 1.024 & 10 & 1.080 & 12 \\
Mechanical brake & 1.011 & 10 & 1.018 & 12 & 1.080 & 11 \\
Main shaft & 1.006 & 12 & 1.020 & 11 & 1.094 & 8 \\
All others & 1.091 & 1 & 1.081 & 5 & 1.287 & 1 \\
\hline
\end{tabular}

The CIMs are evaluated based on wind turbine system failure owing to at least the failure of a subassembly. The subassembly comprising of all other components with CIM $=1.09$ is ranked 1 . However, the electrical subsystem with $\mathrm{CIM}=1.08$ and ranking 2 is to be given the highest maintenance priority among the identified subassemblies to obtain significant improvement in the wind turbine reliability. The main shaft which incidentally has highest reliability is the least critical among the twelve components. As expected, the ranking based on CIM follows the same ordering that is obtained by observing the component failures because a series system conceptualization of failure is considered.

Ranking the subassemblies according to their CAMs, which takes into account their failure rates integrated over failure risk (or consequence) is significantly different from the CIM ranking. The CAMs are computed for a probability of the wind turbine being down for at least 3 days $(\tau=72 \mathrm{~h})$. At this risk level, the highest CAM of 1.24 is recorded for electrical subsystem most probably because the subsystem has the second highest failure rate and the second largest downtime. The failure consequence of this subassembly to the wind turbine system is significant and therefore must be given heightened attention in order to simultaneously reduce system failure and system downtime. The rotor (blades) is the second most critical subassembly for system risk reduction according to its $\mathrm{CAM}=1.12$. The mechanical brake contributes lowest to system unavailability risk since it has the lowest downtime of $48 \mathrm{~h}$ per failure and a relatively low failure rate. The ease in conducting revenue or energy related analysis based on unavailability probabilities makes CAMs better indicators than CIMs for minimizing time, production or revenue losses than CAMs.

CCMs provide the best decision making tools if the objective of stakeholders is to obtain significant reduction in overall annual cost of repairing or restoring system to operation. CCMs are computed using annual probabilities of the upper bound system repair cost to the probability of annual cost of at least $\tau=10,000$ dollars. There is a considerable change to the order to which the subassemblies are ranked using this metric relative to the CIMs or CAMs, as a result of the significant disparities between the repair costs of subassemblies. It is observed that the subassembly containing all other components with $\mathrm{CCM}=1.29$ and the electrical system with $\mathrm{CCM}=1.26$ maintain their first and second positions with regards to importance, respectively, as in the CIM ranking because they have 
high repair costs in addition to their high failure frequencies. However, electrical controls which are ranked third in the CIM have a significantly lower importance according to CCM, occupying the ninth position in the ranking. Another example is the pitch control which is the ninth most critical subassembly according to the CIM and CAM. Owing to its relative contribution to the annual repair cost of the wind turbine, it is regarded as the sixth most valuable unit per CCM.

Even though the three metrics produce different component importance rankings, they still show strong correlation among themselves. The Kendall rank correlation coefficient between CIM and CAM is 0.73 . The correlation coefficient measured between CIM and CCM is 0.64 whereas CAM and CCM are associated by a coefficient of 0.61 . It is worth reiterating that the component failure probabilities used to compute CIMs are also factored in deriving CAMs and CCMs, thus explaining the high correlations between the metrics.

\subsection{Example 2: 45-Component Wind Turbine System}

This example demonstrates the feasibility of realizing an entire set of wind turbine failure event possibilities from the component-level to system-level approach for a large number of components. This approach is needed when failure data of individual components, instead of information on subassembly, are available. Owing to the large number of the components that make up a wind turbine, the recursive solution to the combinatorial approach is employed since the naïve exploration of all possible events becomes computationally impractical. Summaries of wind farm operational data collected by a European consortium of researchers and engineers [25] is used. The field information is 450 wind-farm month's worth of data comprising 350 modern onshore wind turbines. It contains annual failure rates of 62 components expressed as percentage contribution to the average overall failure rate of a wind turbine. Information for supplementary components is excluded in this study, trimming the system size to 45 components. Percentage contributions are multiplied by an overall average failure rate of a system of 1.8 per turbine per year [20] to obtain un-normalized component failure rates.

There are $2^{45}=3.518 \times 10^{13}$ possible $\boldsymbol{k}^{*} \in \boldsymbol{K}$ vectors (or unique combinations) describing system events. This value represents at least the number of operations that would have been required by using the non-recursive combinatorial approach. However, Equation (5) reduces this number to 91,125 computations via the recursive formulation yielding the system failure probability distributions shown in Figure 5. The distribution contains all possibilities of system event. If a series system is considered, this wind turbine system has a reliability of 0.166 . The annual probability of system failure due to at least 2 components is $51 \%$. The likelihood of system failure at the instance of at least 5 components is about $2 \%$. There is an insignificant chance of failure occurrence if system failure is defined by at least 6 out of the 45 components. The recursive approach also provides a reliability bound of 0.32 to 0.83 as a series represented system with positive event correlation. The recursive approach proves to agree perfectly with Monte Carlo simulations (MCS) as shown in Figure 6. 
Figure 5. Annual failure probability distributions of the 45-component wind turbine.

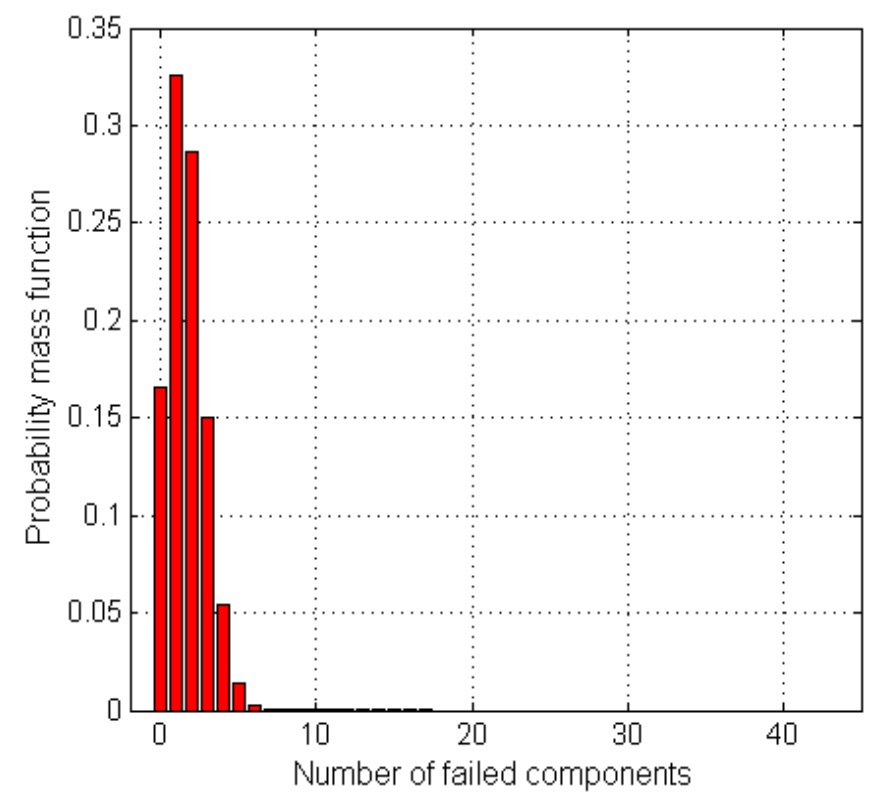

Figure 6. Comparison of annual system failure CDFs obtained for 45-component wind turbine by the recursive solution and a naïve Monte Carlo simulation (MCS) approach.

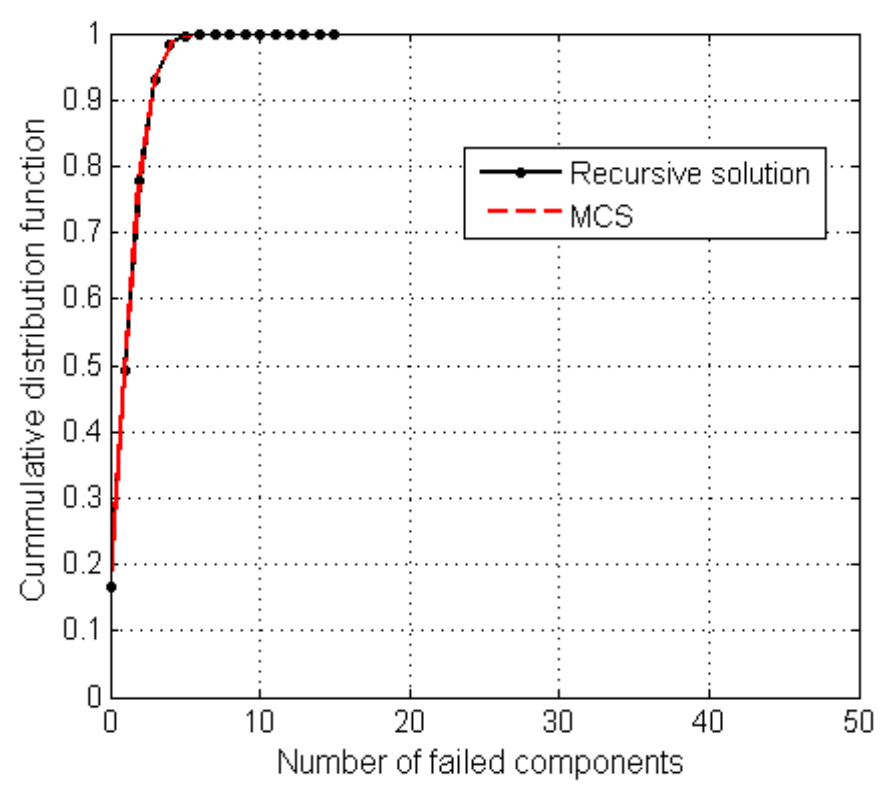

\section{Conclusions}

This paper introduces a closed-form method for assessing the system reliability of wind turbines. The method is capable of explicitly computing the probabilities of all possible configurations of component survivals and failures, and provides an entire distribution of wind turbine failure probability. The flexibility of the combinatorial closed-form approach makes it adaptable for consequence analysis that incorporates unique attributes of turbine component events such as downtime and repair cost. Moreover, the approach enables sensitivity analysis for quantifying the criticality of individual components to wind turbine system reliability. 
The paper illustrates the method by applying it to a 12 -subassembly wind turbine, and evaluating annual probability distributions of system failure as a function of the component failure probabilities. The probability mass function obtained is validated via Monte Carlo simulations. Component failure probabilities are weighted by individual component downtimes per failure to compute annual probabilities of wind turbine unavailability. The computed unavailability risks are readily useful in predicting expected number of stoppages, loss time, power generation and revenue loss per wind turbine in a given year. Furthermore, the costs of repairing, replacing or rebuilding components are also embedded in the combinatorial formulation and a cost-based reliability analysis is performed. Additionally, the paper introduces a component importance metric (CIM) and a component risk metric (CRM), which enhance the information yielded by the closed-form technique. Depending on the empirical information available, wind turbine components may be ranked on the basis of either of the metrics. CIMs are computed on the basis of their survival contribution to the reliability of the wind turbine. CRMs account not only for component failure occurrences but also the system risk implication of component failures in terms of repair cost or downtime. These metrics are useful for resource allocation, maintenance and operational planning, cost minimization and revenue optimization in order to sustain or improve wind turbine performance. Finally, the study uses a recursive solution to the closed form method in order to evaluate failure probabilities of a large 45-component wind turbine system for different failure event definitions. The solution proves to be computationally efficient by performing what would otherwise be $2^{N}$ possible system configurations using a naïve approach in polynomial time $O\left(N^{2}\right)$.

The closed-form method provides a tractable and flexible approach for assessing system reliability and risks of structures whose system events are not well understood. The inherent features of the method demonstrated in this paper highlight the effectiveness of the method as compared to numerical and other analytical techniques for decision-making. The closed-form technique, in its recursive version, also proved to be efficient in handling systems with large numbers of components when calculating its probability mass function of performance, while also providing reliability bounds to handle component correlations. A continuation of this study will focus on improving the recursive solution in order to integrate varying attributes of component failures for risk estimation. An improved version of the recursive approach will facilitate extensive system-risk analysis covering all component-level information. Furthermore, modifications needed in order for the method to explicitly account for correlations between component events characterized by arbitrary probability distributions will also be explored.

\section{Acknowledgments}

This research has been partly funded by the U.S. National Science Foundation through grant CMMI-0748231. Any opinions, findings, and conclusions or recommendations expressed in this material are those of the authors and do not necessarily reflect the views of NSF. The authors also acknowledge the financial contributions from the Department of Civil and Environmental Engineering at Rice University. 


\section{References}

1. Hameed, Z.; Vatn, J.; Heggset, J. Challenges in the reliability and maintainability data collection for offshore wind turbines. Renew. Energy 2011, 36, 2154-2165.

2. 2010 U.S. Wind Industry Market Update, 2011. America Wind Energy Association Web site. Available online: http://www.awea.org/learnabout/publications/ (accessed on 15 August 2011).

3. Guo, H.; Watson, S.; Tavner, P.; Xiang, J. Reliability analysis for wind turbines with incomplete failure data collected from after the date of initial installation. Reliab. Eng. Syst. Saf. 2009, 94, 1057-1063.

4. Song, J.; Kang, W.-H. System reliability and sensitivity under statistical dependence by matrix-based system reliability method. Struct. Saf. 2009, 31, 148-156.

5. Wangdee, W.; Billinton, R. Reliability assessment of bulk electric systems containing large wind farms. Int. J. Electr. Power Energy Syst. 2007, 29, 759-766.

6. Dueñas-Osorio, L.; Craig, J.I.; Goodno, B.J. Seismic response of critical interdependent networks. Earthq. Eng. Struct. Dyn. 2007, 36, 285-306.

7. Adachi, T.; Ellingwood, B.R. Serviceability assessment of a municipal water system under spatially correlated seismic intensities. Comput.-Aided Civ. Infrastruct. Eng. 2009, 24, 237-248.

8. Dueñas-Osorio, L.; Rojo, J. Reliability assessment of lifeline systems with radial topology. Comput.-Aided Civ. Infrastruct. Eng. 2011, 26, 111-128.

9. Duenas-Osorio, L.; Padgett, J.E. Seismic reliability assessment of bridges with user-defined system failure events. J. Eng. Mech. 2011, 137, 680-690.

10. Kang, W.-H.; Song, J.; Gardoni, P. Matrix-based system reliability method and applications to bridge networks. Reliab. Eng. Syst. Saf. 2008, 93, 1584-1593.

11. Karki, R.; Billinton, R. Cost-effective wind energy utilization for reliable power supply. Energy Convers. IEEE Trans. 2004, 19, 435-440.

12. Arabian-Hoseynabadi, H.; Oraee, H.; Tavner, P.J. Wind turbine productivity considering electrical subassembly reliability. Renew. Energy 2010, 35, 190-197.

13. Arabian-Hoseynabadi, H.; Oraee, H.; Tavner, P.J. Failure modes and effects analysis (FMEA) for wind turbines. Int. J. Electr. Power Energy Syst. 2010, 32, 817-824.

14. Sørensen, J.D. Framework for risk-based planning of operation and maintenance for offshore wind turbines. Wind Energy 2009, 12, 493-506.

15. Sørensen, J.D.; Toft, H.S. Probabilistic design of wind turbines. Energies 2010, 3, 241-257.

16. Continuous Reliability Enhancement for Wind (CREW) Database, 2011. Sandia National Laboratories Web site. Available online: http://energy.sandia.gov/?page_id=6682 (accessed on 12 October 2011).

17. U.S. Wind Turbine Reliability Survey Web site. Available online: http://duenas-osorio.rice.edu/ survey/ (accessed on 15 December 2011).

18. Rojo, J.; Dueñas-Osorio, L. Recursive reliability assessment of radial lifeline systems with correlated component failures. In Proceeding of the 11th International Conference on Applications of Statistics and Probability in Civil Engineering (ICASP11), Zurich, Switzerland, 1-4 August, 2011; Faber, M.H., Kohler, J., Nishijima, K., Eds.; CRC Press, Taylor and Francis Group: London, UK, 2011; pp. 1435-1443. 
19. Volkanovski, A.; Čepin, M.; Mavko, B. Application of the fault tree analysis for assessment of power system reliability. Reliab. Eng. Syst. Saf. 2009, 94, 1116-1127.

20. Tavner, P.J.; Xiang, J.; Spinato, F. Reliability analysis for wind turbines. Wind Energy 2007, 10, $1-18$.

21. Eggersgl, W. Wind Energie; Landwirtschaftskammer Schleswig-Holstein: Rendsburg, Germany, 1993-2004; Volume V-XVI.

22. Spinato, F. The Reliability of Wind Turbines; Durham University: Durham, UK, 2008.

23. Poore, R.; Walford, C. Development of an Operations and Maintenance Cost Model to Identify Cost of Energy Savings for Low Wind Speed Turbines; National Renewable Energy Laboratory: Seattle, WA, USA, 2008. Available online: http://www.nrel.gov/docs/fy08osti/40581.pdf (accessed on 22 January 2012 ).

24. Nielsen, J.J.; Sørensen, J.D. On risk-based operation and maintenance of offshore wind turbine components. Reliab. Eng. Syst. Saf. 2011, 96, 218-229.

25. Reliawind Reliability Focused Research on Optimizing Wind Energy Systems Design, Operation and Maintenance: Tools, Proof of Concepts, Guidelines \& Methodologies for a New Generation, 2011. Relia Wind Project Web site. Available online: http://www.reliawind.eu/files/file-inline/ (accessed on 2 September 2011).

(C) 2012 by the authors; licensee MDPI, Basel, Switzerland. This article is an open access article distributed under the terms and conditions of the Creative Commons Attribution license (http://creativecommons.org/licenses/by/3.0/). 